\title{
COMMUTATION RELATIONS AND HYPERCYCLIC OPERATORS
}

\author{
VITALY E. KIM
}

\begin{abstract}
In this paper we establish hypercyclicity of continuous linear operators on $H(\mathbb{C})$ that satisfy certain commutation relations.
\end{abstract}

\section{Introduction and statement of the main results}

Let $X$ be a topological vector space. A continuous linear operator $T: X \rightarrow X$ is said to be hypercyclic if there exists an element $x \in X$ such that it's orbit $\operatorname{Orb}(T, x)=\left\{T^{n} x, n=\right.$ $0,1,2, \ldots\}$ is dense in $X$. Let us denote by $H(\mathbb{C})$ the space of all entire functions with the uniform convergence topology. For $\lambda \in \mathbb{C}$ we will denote by $S_{\lambda}$ the translation operator on $H(\mathbb{C}): S_{\lambda} f(z) \equiv f(z+\lambda)$. The first examples of hypercyclic operators are due to $\mathrm{G}$. D. Birkhoff and G. MacLane. In 1929, Birkhoff [4] showed that the translation operator $S_{\lambda}$ is hypercyclic on $H(\mathbb{C})$ if $\lambda \neq 0$. In 1952, MacLane [14] proved that the differentiation operator is hypercyclic on $H(\mathbb{C})$. A significant generalization of these results was proved by G. Godefroy and J. H. Shapiro [8] in 1991. They showed that every convolution operator on $H\left(\mathbb{C}^{N}\right)$ that is not a scalar multiple of the identity is hypercyclic. Recall that a convolution operator on $H\left(\mathbb{C}^{N}\right)$ is a continuous linear operator that commutes with all translations (or equivalently, commutes with each partial differentiation operator).

Some classes of non-convolution hypercyclic operators on $H(\mathbb{C})\left(\right.$ or $\left.H\left(\mathbb{C}^{N}\right)\right)$ can be found in [1], [6], [15], [3], [12]. More information on hypercyclic operators can be found in [9], [2], [10].

In the present paper we prove the following result.

Theorem 1.1. Let $T: H(\mathbb{C}) \rightarrow H(\mathbb{C})$ be a continuous linear operator that satisfies the following conditions:

1. $\operatorname{ker} T \neq\{0\}$ 
2. T satisfies the commutation relation:

$$
\left[T, \frac{\mathrm{d}}{\mathrm{d} z}\right]=a I
$$

where $a \in \mathbb{C} \backslash\{0\}$ is some constant and $I$ is identity operator.

Then $T$ is hypercyclic.

Remark 1.2. In Theorem 1.1 we consider a constant $a \neq 0$. Note that if $a=0$ in (1) then $T$ is a convolution operator on $H(\mathbb{C})$ that is hypercyclic by a Theorem of Godefroy and Shapiro [8].

We also prove the following more general result.

Theorem 1.3. Let $T: H(\mathbb{C}) \rightarrow H(\mathbb{C})$ satisfies the conditions of Theorem 1.1 and let $L$ be an entire function such that $L \not \equiv$ const and $L(T)$ is a continuous operator from $H(\mathbb{C})$ to itself. Then $L(T)$ is hypercyclic.

The paper is organised as follows. In Section 2 we formulate and prove a theorem that establishes the completeness of translates of certain entire functions. In Section 3 we prove Theorems 1.1 and 1.3 by application of the results of Section 2 and the GodefroyShapiro criterion. In Section 4 we give a description of operators that are hypercyclic by Theorem 1.1 or 1.3 and provide some examples.

\section{Complete systems of translates of entire functions}

Recall that the system of entire functions $\left\{f_{\lambda}(z), \lambda \in \Lambda \subset \mathbb{C}\right\}$ is called complete in $H(\mathbb{C})$ if $\overline{\operatorname{span}\left\{f_{\lambda}, \lambda \in \Lambda\right\}}=H(\mathbb{C})$. The connection between complete systems of entire functions and hypercyclic operators on $H(\mathbb{C})$ is established by the Godefroy-Shapiro criterion which was exhibited by G. Godefroy and J. H. Shapiro in [8] (see also e.g. [2, Ch. 1]):

Theorem 2.1 (Godefroy-Shapiro criterion). Let $T$ be a continuous linear operator on $H(\mathbb{C})$. Suppose that there is a system of entire functions $\left\{f_{\lambda}\right\}_{\lambda \in \mathbb{C}}$ such that:

1. $T\left[f_{\lambda}\right]=\lambda f_{\lambda}, \forall \lambda \in \mathbb{C}$; 
2. both systems $\left\{f_{\lambda}, \lambda \in \mathbb{C}:|\lambda|<1\right\}$ and $\left\{f_{\lambda}, \lambda \in \mathbb{C}:|\lambda|>1\right\}$ are complete in $H(\mathbb{C})$. Then $T$ is hypercyclic

In this section we prove the following result.

Theorem 2.2. Let $T: H(\mathbb{C}) \rightarrow H(\mathbb{C})$ satisfies the conditions of Theorem 1.1 for some $a \neq 0$. Then the system $\left\{S_{\lambda} f\right\}_{\lambda \in \Lambda}$ is complete in $H(\mathbb{C})$ for any function $f \in \operatorname{ker} T \backslash\{0\}$ and any set $\Lambda \subset \mathbb{C}$ that has an accumulation point in $\mathbb{C}$.

Proof. Let $T$ satisfy the conditions of Theorem 1.1. Then there is an entire function $f$ such that $f \in \operatorname{ker} T$ and $f \not \equiv 0$. From (1) it follows that

$$
\left[T, \frac{\mathrm{d}^{n}}{\mathrm{~d} z^{n}}\right]=\operatorname{an} \frac{\mathrm{d}^{n-1}}{\mathrm{~d} z^{n-1}}, n=0,1, \ldots
$$

(see e.g. [5, §16]). Hence,

$$
T f^{(n)}=a n f^{(n-1)}, n=0,1, \ldots
$$

Recall that every convolution operator $M_{L}$ on $H(\mathbb{C})$ can be represented as a linear differential operator of, generally speaking, infinite order with constant coefficients:

$$
M_{L}[f](z)=\sum_{k=0}^{\infty} d_{k} f^{(k)}(z),
$$

where $L(\lambda)=\sum_{k=0}^{\infty} d_{k} \lambda^{k}$ is an entire function of exponential type. The function $L$ is called a characteristic function of the convolution operator (3). A convolution operator (3) can be also represented in the form

$$
M_{L}[f](\lambda)=\left(F_{L}, S_{\lambda} f\right)
$$

where $F_{L}$ is a continuous linear functional on $H(\mathbb{C})$ such that $\left(F_{L}, \exp (\lambda z)\right)=L(\lambda)$ (see e.g. [13]).

Let $\left\{\lambda_{j}\right\}_{j=1}^{\infty}$ be the set of zeros of $L$. Denote by $m_{j}$ the multiplicity of zero $\lambda_{j}$. Then ker $M_{L}$ contains exponential monomials $z^{b} \exp \left(\lambda_{j} z\right), b=0,1, \ldots, m_{j}-1, j \in \mathbb{N}$ and does not contain any other exponential monomials. In particular, ker $M_{L}$ does not contain any 
exponential monomial of the kind $z^{p} \exp (\mu z)$ if $L(\mu) \neq 0$. By the result of L. Schwartz [16], every differentiation-invariant closed linear subspace $V$ of $H(\mathbb{C})$ coincides with the closure of the linear span of exponential monomials that are contained in $V$. Hence,

$$
\operatorname{ker} M_{L}=\overline{\operatorname{span}\left\{z^{b} \exp \left(\lambda_{j} z\right), j \in \mathbb{N}, b=0,1, \ldots, m_{j}-1\right\}} \text {. }
$$

Denote by $\operatorname{Conv}[H(\mathbb{C})]$ the class of all convolution operators (44) on $H(\mathbb{C})$ except the operator of multiplication by zero.

Let $\Lambda$ be an arbitrary subset of $\mathbb{C}$ that has an accumulation point in $\mathbb{C}$. In order to prove that the system $\left\{S_{\lambda} f\right\}_{\lambda \in \Lambda}$ is complete in $H(\mathbb{C})$, it is enough to prove that $f \notin \operatorname{ker} M$ for any $M \in \operatorname{Conv}[H(\mathbb{C})]$. Indeed, if the system $\left\{S_{\lambda} f\right\}_{\lambda \in \Lambda}$ is not complete in $H(\mathbb{C})$, then according to the Approximation Principle (see e.g. [7, Ch. 2]) there is a non-zero continuous linear functional $F$ on $H(\mathbb{C})$ such that $\left(F, S_{\lambda} f\right)=0, \forall \lambda \in \Lambda$ and hence, $f \in \operatorname{ker} M$ for some $M \in \operatorname{Conv}[H(\mathbb{C})]$.

Assume that $f \in \operatorname{ker} M_{\Phi}$ for some $M_{\Phi} \in \operatorname{Conv}[H(\mathbb{C})]$ with the characteristic function $\Phi(\lambda)=\sum_{k=0}^{\infty} b_{k} \lambda^{k}$, i.e.

$$
\sum_{k=0}^{\infty} b_{k} f^{(k)}(z) \equiv 0 .
$$

Then, obviously, $T^{n} M_{\Phi} f=0, \forall n \in \mathbb{N}$. Using (2) we obtain

$$
T^{n} M_{\Phi}[f](z) \equiv a^{n} \sum_{k=n}^{\infty} b_{k} \frac{k !}{(k-n) !} f^{(k-n)}(z) .
$$

Hence,

$$
\sum_{k=n}^{\infty} b_{k} \frac{k !}{(k-n) !} f^{(k-n)}(z) \equiv 0, n=0,1, \cdots .
$$

We see that $f$ satisfies the infinite system of convolution equations (5) with the characteristic functions $\Phi^{(n)}, n=0,1, \cdots$. Let us note that $\Phi \not \equiv 0$, since by assumption $M_{\Phi} \in \operatorname{Conv}[H(\mathbb{C})]$.

Denote $W=\bigcap_{n=0}^{\infty} \operatorname{ker} M_{\Phi^{(n)}}$. It is easy to see that $W$ is differentiation-invariant closed linear subspace of $H(\mathbb{C})$. Assume that $W$ contains some exponential monomial of the kind $z^{p} \exp (\mu z)$. Then $\Phi^{(n)}(\mu)=0, n=0,1, \cdots$. But this is impossible, since by assumption $\Phi \not \equiv 0$. Hence, $W$ does not contain any functions of the kind $z^{p} \exp (\mu z)$. Then, by the 
result of L. Schwartz [16], $W$ is trivial, i.e. $W=\{0\}$. We have a contradiciton to the fact that $f \in W$ and $f \not \equiv 0$. Hence, $f \notin \operatorname{ker} M_{\Phi}$.

Remark 2.3. In the proof of Theorem [2.2 we use the result of L. Schwartz [16] on spectral synthesis in $H(\mathbb{C})$. But we cannot prove the analogue of Theorem 2.2 for $H\left(\mathbb{C}^{N}\right)$ $(N>1)$ using the same method because there is a conter-example on spectral synthesis in $H\left(\mathbb{C}^{N}\right)$ by D. Gurevich [11]. In particular Gurevich showed that there is a non-trivial differentiation-invariant subspace $V$ in $H\left(\mathbb{C}^{N}\right)$ that does not contain any exponential monomial. We are going to discuss the analogues of Theorems [2.2. 1.1 and 1.3 for $H\left(\mathbb{C}^{N}\right)$ in one of the next papers.

\section{Proofs of Theorems 1.1 and 1.3}

It is enough to prove Theorem 1.3, since Theorem 1.1 is a special case of Theorem 1.3 , when $L(z) \equiv z$. Let $T: H(\mathbb{C}) \rightarrow H(\mathbb{C})$ and $L \in H(\mathbb{C})$ satisfy the conditions of Theorem 1.3. Let $f \in \operatorname{ker} T \backslash\{0\}$. Using the Taylor expansion we can write:

$$
S_{\lambda} f(z)=\sum_{n=0}^{\infty} \frac{f^{(n)}(z)}{n !} \lambda^{n} .
$$

Then from (2) it follows that

$$
T S_{\lambda} f=\sum_{n=0}^{\infty} \frac{T\left[f^{(n)}(z)\right]}{n !} \lambda^{n}=\sum_{n=1}^{\infty} \frac{a n f^{(n-1)}(z)}{n !} \lambda^{n}=a \lambda \sum_{n=0}^{\infty} \frac{f^{(n)}(z)}{n !} \lambda^{n} .
$$

Hence, $T S_{\lambda} f=a \lambda S_{\lambda} f$ and $L(T) f=L_{a}(\lambda) S_{\lambda} f, \forall \lambda \in \mathbb{C}$, where $L_{a}(\lambda) \equiv L(a \lambda)$. Since $L_{a} \not \equiv$ const, then both sets $\left\{\lambda \in \mathbb{C}:\left|L_{a}(\lambda)\right|<1\right\}$ and $\left\{\lambda \in \mathbb{C}:\left|L_{a}(\lambda)\right|>1\right\}$ are non-empty open subsets of $\mathbb{C}$. Hence, $L(T)$ is hypercyclic by Theorems 2.2 and 2.1 .

\section{Operators that satisfy Theorem 1.1 or 1.3}

In this section we give the examples of linear continuous operators on $H(\mathbb{C})$ which are hypercyclic by Theorem 1.1 or 1.3. First, we shall describe the class of linear continuous operators on $H(\mathbb{C})$ that satisfy the condition 2 of Theorem 1.1 . 
Theorem 4.1. A continuous linear operator $T: H(\mathbb{C}) \rightarrow H(\mathbb{C})$ satisfies the commutation relations (1) if and only if $T=M-a z I$, where $M$ is a convolution operator (3) on $H(\mathbb{C})$.

Proof. 1) Let $T=M-a z I$, where $M$ is an arbitrary convolution operator $(3)$ on $H(\mathbb{C})$. Then we have $\left[T, \frac{\mathrm{d}}{\mathrm{d} z}\right]=\left[M, \frac{\mathrm{d}}{\mathrm{d} z}\right]-\left[a z I, \frac{\mathrm{d}}{\mathrm{d} z}\right]=a I$.

2) Let $T$ satisfies the commutation relation (11), i.e. $\left[T, \frac{\mathrm{d}}{\mathrm{d} z}\right]=a I$. Let us define the operator $T_{0}=-a z I$. Then $\left[T_{0}, \frac{\mathrm{d}}{\mathrm{d} z}\right]=a I$. Denote $M=T-T_{0}$. Then $\left[M, \frac{\mathrm{d}}{\mathrm{d} z}\right]=[T-$ $\left.T_{0}, \frac{\mathrm{d}}{\mathrm{d} z}\right]=0$. Hence, $M$ is a convolution operator on $H(\mathbb{C})$, and $T=T_{0}+M=M-a z I$.

Let $T=M-a z I$, where $M$ is a convolution operator. Then $T$ is hypercyclic by Theorems 1.1 and 4.1 if there exists an entire function $f$ such that $f \not \equiv 0$ and $f \in$ $\operatorname{ker} T$. Here we provide some examples of such functions and corresponding hypercyclic operators.

Example a) Let $T=\frac{d}{d z}-z I$. If $f(z)=C e^{z^{2} / 2}$, where $C$ is some constant, then $f \in \operatorname{ker} T$. Hence, $\mathrm{T}$ is hypercyclic by Theorem 1.1. Consider the operator

$$
L(T)=\frac{d^{2}}{d z^{2}}+(2 z-1) \frac{d}{d z}+\left(z^{2}-z-1\right) I,
$$

where $L(\lambda)=\lambda^{2}+\lambda$. Then the operator (6) is hypercyclic by Theorem 1.3 ,

b) Let $T=\frac{d^{2}}{d z^{2}}-z I$. If $f(z)=C_{1} \operatorname{Ai}(z)+C_{2} \operatorname{Bi}(z)$, where $C_{1}, C_{2}$ are some constants, and $\mathrm{Ai}, \mathrm{Bi}$ are Airy functions (see e.g. [17]), then $f \in \operatorname{ker} T$. Hence, T is hypercyclic by Theorem 1.1.

Now we will describe the class of hypercyclic differential operators of infinite order with polynomial coefficients. This class contains as a partial case all convolution operators on $H(\mathbb{C})$ that are not scalar multiples of the identity.

Theorem 4.2. Let $T=\frac{d}{d z}-a z I$, where $a \in \mathbb{C}$ is some constant. Let $L(z)=\sum_{k=0}^{\infty} d_{k} \lambda^{k}$ be an arbitrary entire function of exponential type such that $L \not \equiv$ const. Then the operator

$$
L(T)=\sum_{k=0}^{\infty} d_{k} T^{k}
$$

is hypercyclic operator on $H(\mathbb{C})$. 
Proof. Let's show that $L(T) f \in H(\mathbb{C}), \forall f \in H(\mathbb{C})$. We can represent $f$ in the form $f(z)=e^{a z^{2} / 2} g(z)$. Then $T^{k} f(z)=e^{a z^{2} / 2} g^{(k)}(z), k=0,1, \cdots$. Hence, $L(T) f(z)=$ $e^{a z^{2} / 2} M_{L} g(z) \in H(\mathbb{C})$, where $M_{L}$ is a convolution operator on $H(\mathbb{C})$ generated by $L$. Since $T$ satisfies the conditions of Theorem 1.1, then $L(T)$ is hypercyclic by Theorem 1.3

\section{References}

[1] R. Aron, D. Markose, On universal functions. J. Korean Math. Soc. 41:1 (2004), $65-76$

[2] F. Bayart, E. Matheron, Dynamics of linear operators. Cambridge University Press, 2009.

[3] J. J. Betancor, M. Sifi, K. Trimeche, Hypercyclic and chaotic convolution operators associated with the Dunkl operators on $\mathbb{C}$. Acta Math. Hungar. 106 (2005), 101-116.

[4] G. D. Birkhoff, Demonstration d'un theoreme elementaire sur les fonctions entieres. C. R. Acad. Sci. Paris 189 (1929), 473-475.

[5] L. D. Faddeev, O. A. Yakubovskii, Lectures on Quantum Mechanics for Mathematics Students. AMS, 2009.

[6] G. Fernandez, A. A. Hallack, Remarks on a result about hypercyclic non-convolution operators. J. Math. Anal. Appl. 309:1 (2005), 52-55.

[7] R. E. Edwards, Functional analysis: theory and applications. Dover Publications, 1995.

[8] G. Godefroy, J. H. Shapiro, Operators with dense, invariant, cyclic vector manifolds. J. Funct. Anal. 98:2 (1991), 229-269.

[9] K.-G. Grosse-Erdmann, Universal families and hypercyclic operators. Bull. Amer. Math. Soc. 36:3 (1999), 345-381. 
[10] K.-G. Grosse-Erdmann, A. Peris Manguillot, Linear chaos. Springer, 2011.

[11] D. I. Gurevich, Counterexamples to a problem of L. Schwartz (in Russian). Funkts. Anal. Prilozh. 9:2 (1975), 29-35 [English translation: Funct. Anal. Appl. 9:2 (1975), 116-120].

[12] V. E. Kim, Hypercyclicity and chaotic character of generalized convolution operators generated by Gelfond-Leontev operators (in Russian). Mat. Zametki 85:6 (2009), 849856 [English translation: Math. Notes 85:6 (2009), 807-813].

[13] A. S. Krivosheev, V. V. Napalkov, Complex analysis and convolution operators (in Russian). Usp. Mat. Nauk 47:6 (1992), 3-58 [English translation: Russ. Math. Surv. 47:6 (1992), 1-56].

[14] G. MacLane, Sequences of derivatives and normal families. J. Anal. Math. 2 (1952), $72-87$.

[15] H. Petersson, Supercyclic and hypercyclic non-convolution operators. J. Operator Theory 55:1 (2006), 133-151.

[16] L. Schwartz, Theorie generale des fonctions moyenne-periodique. Ann. Math. 48:4 (1947), 867-929.

[17] O. Vallee, M. Soares, Airy functions and applications to physics. Imperial College Press, 2004.

Vitaly E. Kim

Institute of Mathematics with Computing Centre,

Russian Academy of Sciences,

112 Chernyshevsky str.,

450008 Ufa, Russia

e-mail: kim@matem.anrb.ru 